Subscriber access provided by Kaohsiung Medical University

\title{
Communication
}

\section{NanoSQUID Magnetometry on Individual As-grown and Annealed Co Nanowires at Variable Temperature}

Maria Jose Martinez-Perez, Javier Pablo-Navarro, Benedikt Mueller, Reinhold Kleiner, Cesar Magen, Dieter Koelle, Jose Maria De Teresa, and Javier Sesé

Nano Lett., Just Accepted Manuscript • DOI: 10.1021/acs.nanolett.8b03329 • Publication Date (Web): 20 Nov 2018

Downloaded from http://pubs.acs.org on November 21, 2018

\section{Just Accepted}

"Just Accepted" manuscripts have been peer-reviewed and accepted for publication. They are posted online prior to technical editing, formatting for publication and author proofing. The American Chemical Society provides "Just Accepted" as a service to the research community to expedite the dissemination of scientific material as soon as possible after acceptance. "Just Accepted" manuscripts appear in full in PDF format accompanied by an HTML abstract. "Just Accepted" manuscripts have been fully peer reviewed, but should not be considered the official version of record. They are citable by the Digital Object Identifier (DOI®). "Just Accepted" is an optional service offered to authors. Therefore, the "Just Accepted" Web site may not include all articles that will be published in the journal. After a manuscript is technically edited and formatted, it will be removed from the "Just Accepted" Web site and published as an ASAP article. Note that technical editing may introduce minor changes to the manuscript text and/or graphics which could affect content, and all legal disclaimers and ethical guidelines that apply to the journal pertain. ACS cannot be held responsible for errors or consequences arising from the use of information contained in these "Just Accepted" manuscripts. 


\title{
NanoSQUID Magnetometry on Individual
}

\section{As-grown and Annealed Co Nanowires at Variable Temperature.}

\author{
M. J. Martínez-Pérez, ${ }^{*, \dagger, \ddagger ~ J . ~ P a b l o-N a v a r r o, ~}{ }^{\Uparrow}$ B. Müller, ${ }^{\S}$ R. Kleiner, ${ }^{\S}$ C. \\ Magén, ${ }^{\dagger} \boldsymbol{\oplus}$ D. Koelle, ${ }^{\S}$ J. M. de Teresa, ${ }^{\dagger,}$ and J. Sesét, \\ $\dagger$ Instituto de Ciencia de Materiales de Aragón and Departamento de Física de la Materia \\ Condensada, CSIC-Universidad de Zaragoza, 50009 Zaragoza, Spain \\ $\ddagger$ Fundación ARAID, 50018 Zaragoza, Spain \\ \Laboratorio de Microscopías Avanzadas (LMA), Instituto de Nanociencia de Aragón \\ (INA), Universidad de Zaragoza, 50018 Zaragoza, Spain \\ $\S$ Physikalisches Institut - Experimentalphysik II and Center for Quantum Science (CQ) in \\ LISA+ ${ }^{+}$, Universität Tübingen, 72076 Tübingen, Germany \\ E-mail: pemar@unizar.es
}

\begin{abstract}
Performing magnetization studies on individual nanoparticles is a highly demanding task, especially when measurements need to be carried out under large sweeping magnetic fields or variable temperature. Yet, characterization under varying ambient conditions is paramount in order to fully understand the magnetic behavior of these objects, e.g., the formation of non-uniform states or the mechanisms leading to magnetization reversal and thermal stability. This, in turn, is necessary for the integration of magnetic nanoparticles and nanowires into useful devices, e.g., spin-valves, racetrack
\end{abstract}


memories or magnetic tip probes. Here we show that nano-superconducting quantum interference devices based on high critical temperature superconductors are particularly well suited for this task. We have successfully characterized a number of individual Co nanowires grown through focused electron beam induced deposition and subsequently annealed at different temperatures. Magnetization measurements performed under sweeping magnetic fields (up to $\sim 100 \mathrm{mT}$ ) and variable temperature $(1.4-80$ K) underscore the intrinsic structural and chemical differences between these nanowires. These point to significant changes in the crystalline structure and the resulting effective magnetic anisotropy of the nanowires, and to the nucleation and subsequent vanishing of antiferromagnetic species within the nanowires annealed at different temperatures.

\section{Keywords}

NanoSQUID, magnetization measurements, magnetic nanowires, focused electron beam induced deposition, magnetization reversal

\section{Introduction}

Beyond well-studied two-dimensional (stripe-like) planar magnetic nanowires, the scientific community is increasingly interested in their three-dimensional (3D) counterparts. ${ }^{1}$ Not being restricted to the plane, 3D nanowires (NWs) offer new functionalities with potential for applications such as vertical racetrack memories, ${ }^{2}$ magnetic logic, ${ }^{3}$ magnetic nano-cantilevers for high-resolution imaging ${ }^{4}$ or actuators. ${ }^{5}$ Due to their geometry, these objects also offer a richer variety of available magnetic configurations and possible domain walls with very attractive topological and dynamical properties that are appealing from a fundamental and technological point of view. ${ }^{6,7}$

Magnetic characterization of such small objects still poses many technological challenges in terms of spin sensitivity and operation conditions, i.e., the possibility of applying large 

(MOKE) microscopy, Hall sensors and off-axis electron holography have been successfully applied to the study of 3D magnetic NWs. ${ }^{8-11}$ However, MOKE does not usually offer the possibility of working under variable temperature and provides very poor sensitivity. Hall sensors, on the other hand, are noted for operating in extraordinary large magnetic field and temperature ranges being, however, not well-suited for ultra-sensitive sensing. Electron holography is characterized by its capability to produce spatially resolved quantitative imaging of the magnetization states as a function of temperature and magnetic field, ${ }^{12}$ even tomographic. ${ }^{13}$ However it has also limited sensitivity and the stability requirements impose remarkable experimental constraints. More recently, ultra-soft oscillating microcantilevers have been operated as force sensors to detect individual magnetic NWs. ${ }^{14}$ The sensitivity can reach impressive values of few Bohr magnetons when using cantilevers made of, e.g., carbon nanotubes. ${ }^{15}$ Such an approach is, however, technologically very challenging and the results can not be interpreted straightforwardly. Alternatively, nano-Superconducting Quantum Interference Devices (nanoSQUIDs) provide a direct measure of minute fractions of the magnetic flux quantum $\left(\Phi_{0}\right) \cdot{ }^{16-19}$ These sensors were indeed used in the first pioneering experiments for detecting individual magnetic nanoparticles and NWs. ${ }^{20,21}$ Being superconducting, nanoSQUIDs are usually restricted to narrow temperature and field ranges. We have beaten such limitations by using the high critical temperature and high critical field superconductor $\mathrm{YBa}_{2} \mathrm{Cu}_{3} \mathrm{O}_{7}$ (YBCO) and grain boundary Josephson junctions. ${ }^{22,23}$ The resulting nanoSQUIDs are fully operative upon large in-plane applied magnetic fields (up to $\left.\mu_{0} H=1 \mathrm{~T}\right)$ and broad temperature ranges $(\mathrm{mK}<T<80 \mathrm{~K}) .{ }^{24}$

Regarding fabrication, 3D NWs can be synthesized using both chemical and physical methods. In the former case, templates such as, e.g., alumina matrices, ${ }^{25}$ carbon nanotubes ${ }^{10}$ or GaAs NWs ${ }^{14}$ are required to produce vertical NWs and hollow nanotubes. Among physical methods, Focused Electron Beam Induced Deposition (FEBID) stands out as it allows depositing 3D nanoscopic objects with arbitrary shape and dimensions down to few tens of 
$\mathrm{nm}$ in a single step. ${ }^{26}$ Unfortunately, Co- and Fe-FEBID magnetic deposits are often formed by nanocrystals with poor metallic content due to the presence of carbonaceous impurities. This yields degraded magnetic and transport properties compared with pure bulk materials. Recently, high-quality Co-FEBID NWs have been obtained by implementing an ex-situ annealing process in high vacuum conditions. ${ }^{27}$ Increasing the annealing temperature up to $600{ }^{\circ} \mathrm{C}$ improves the degree of crystallinity, increasing also the Co purity and saturation magnetization up to values very close to bulk crystalline Co.

Here we present a thorough study of individual Co-FEBID NWs annealed at different temperatures by using YBCO nanoSQUID sensors. For this purpose we have first developed an extremely precise technical protocol that has allowed the growth, annealing and subsequent integration of individual Co-FEBID NWs onto nanoSQUIDs with nanometric resolution. Thanks to the latter, we achieve excellent sample-sensor magnetic coupling. This fact, together with the high spin-sensitivity of YBCO nanoSQUIDs, allows us distinguishing minute magnetic signals produced by domain wall nucleation, pinning/depinning or complete magnetization reversal.

As demonstrated in Ref. 27, NWs annealed at different temperatures undergo drastic structural and chemical changes. These changes have dramatic consequences on the magnetic response of the NWs that become only apparent when performing magnetization measurements at variable temperature. We have succeeded in observing such effects thanks to the broad operation temperature range of YBCO nanoSQUIDs. Based on the observed temperature-dependent magnetization switching of the NWs, we have classified them into three groups. The behavior of the as-grown NW reveals the existence of many defects or impurities and, more importantly, large spin frustration. NWs annealed at 150 and $300{ }^{\circ} \mathrm{C}$ seem to be influenced by the presence of sizable antiferromagnetic crystals. These effects diminish in NWs annealed at 450 and $600{ }^{\circ} \mathrm{C}$ which exhibit higher crystal and chemical quality and increased anisotropy. 


\section{Results}

Experimental setup. 3D Co-FEBID NWs are fabricated by FEBID in a dual-beam system equipped with an electron and a Ga Focused Ion Beam (FIB) column, using $\mathrm{Co}_{2}(\mathrm{CO})_{8}$ precursor gas (see Methods section). Fabrication parameters are chosen to maximize the resulting Co purity while preserving good spatial resolution. ${ }^{28}$ One NW is left as-grown (see Fig. 1a) whereas the rest are annealed at different temperatures in high-vacuum conditions as described in the Methods section. As demonstrated in Ref. 28, all NWs consist of an inner magnetic Co core surrounded by a $\sim 5$ nm-thick non-magnetic layer. Resulting geometrical parameters of all NWs can be seen in Table 1. The as-grown NW and those annealed at 150, 300,450 , and $600{ }^{\circ} \mathrm{C}$ are denoted as RT-, 150-, 300-, 450-, and 600-NW, respectively.

A Scanning Electron Microscopy (SEM) image of a typical nanoSQUID device can be seen in Fig. 1b (see Methods section for details). These devices are operated in a variable temperature insert (minimum temperature $\sim 1.4 \mathrm{~K}$ ) equipped with a rotator that allows aligning the nanoSQUID with respect to the externally applied magnetic field (indicated by a yellow arrow in Fig. 1b). Field must be aligned parallel to the SQUID substrate and perpendicularly to the grain boundary's plane so that no magnetic flux is coupled both to the nanoloop and to the Josephson junctions. Magnetization measurements are performed by sweeping the external magnetic field and measuring the total flux $\Phi$ captured by the nanoSQUID loop as the NW is driven through magnetization reversal.

NWs are transported using an Omniprobe nanomanipulator installed in the dual-beam system. Here, we describe briefly the transport protocol used for the annealed NWs (see the Supporting Information for more details on the transport of RT-NW). Thin (few $\mu$ mthick) $\mathrm{Cu}$ lamellae where NWs have been grown and annealed are used as carriers. The nanomanipulator tip is attached to the corresponding $\mathrm{Cu}$ lamella by Focused Ion Beam Induced Deposition (FIBID) of Pt using $\mathrm{CH}_{3} \mathrm{CpPt}\left(\mathrm{CH}_{3}\right)_{3}$ precursor gas (see Fig. 1c left). The carrier is then located with nanometric resolution over the sensor surface so that the corresponding NW's long axis is parallel to the externally applied magnetic field with the 
end tip lying close to the nanoloop (see Fig. 1c right). A second Pt-FIBID deposit is used to fix the carrier to the substrate. The nanomanipulator tip is finally released by FIB-cutting.

After the transport process, all NWs lie at equivalent positions chosen so to guarantee an optimum NW-sensor coupling (see Fig. 2a). For this purpose, the NWs' end tip lies at just $x \sim 100-300 \mathrm{~nm}$ from the nanoloop (see Fig. 1c, right panel). The corresponding vertical distance $z$ measured from the NWs' end tip to the surface of the sensor is given in Table 1.

Hysteresis loops. Fig. 2b shows representative hysteresis loops measured at $15 \mathrm{~K}$ for each NW (five curves are shown in each panel). Loops are square-shaped indicating that the NWs are in the quasi single-domain state (as expected due to their large aspect-ratio) and that the field is applied along the NW's easy axis. In the classical model of StonerWohlfarth, ${ }^{29}$ the magnetization reversal of a single domain particle takes place coherently, i.e., all magnetic moments reverse at unison. This is only true for very small magnetic objects (few $\mathrm{nm}$ ). More conveniently, magnetic moments will tilt around the easy axis of the NW in a vortex-like configuration which saves some magnetostatic energy at the cost of exchange energy (curling model). ${ }^{30}$ This is a quite accepted mechanism for magnetization reversal in NWs that will serve us as starting point to analyze our experimental data.

Under these circumstances and, assuming a negligible contribution of the magnetocrystalline anisotropy due to the standard polycrystalline nature of the NWs, the nucleation magnetic field is given by $H_{\mathrm{n}}^{\text {cur }}=\kappa M_{\mathrm{s}}\left(\lambda_{\mathrm{ex}} / R\right)^{2} \cdot{ }^{30}$ Here, the exchange length $\lambda_{\mathrm{ex}}$ is defined as $\sqrt{2 A / \mu_{0} M_{\mathrm{s}}^{2}}, \mu_{0}$ being the magnetic permeability constant, $M_{\mathrm{s}}$ the saturation magnetization, $A$ the exchange stiffness, $R$ the radius of the NW and $\kappa=3.39$ for an infinite cylinder. We will approximate $M_{\mathrm{s}}=p \times M_{\mathrm{s}}($ bulk) and $A=p \times A$ (bulk), with $p$ being the Co purity $(0<p<1)$, and $M_{\mathrm{s}}(\mathrm{bulk})=1.4 \times 10^{6} \mathrm{~A} / \mathrm{m}$ and $A(\mathrm{bulk})=2.5 \times 10^{-11} \mathrm{~J} / \mathrm{m}$ for pure crystalline Co. We highlight that, within this very simple model, the resulting values of $H_{\mathrm{n}}^{\text {cur }}$ are independent of $p$ and $l$, depending only on the radius of the NW as $H_{\mathrm{n}}^{\text {cur }} \propto 1 / R^{2}$. Inserting the $R$ values listed in Table 1 yields the values indicated in Fig. $2 \mathbf{b}$ with dashed lines.

In the case of RT- and 150-NW, the curling model overestimates by far the experimentally 
measured nucleation fields. This is usually found in practice as, in real NWs, magnetization reversal is more likely to undergo a nucleation and propagation process. Such a process is energetically favorable and takes place at fields much lower than $H_{\mathrm{n}}^{\text {cur }}$. In this scenario, a small reversed region is first nucleated by curling together with the corresponding domain wall. ${ }^{31}$ Subsequently, this domain wall moves rapidly (ns - ps timescales) through the NW until the magnetization reversal is complete. Strikingly, in the case of 300-, 450- and 600NW, the $H_{\mathrm{n}}^{\text {cur }}$ values are slightly underestimated. This suggests some additional structural differences between the samples annealed at higher temperatures as also supported by the evident differences in the hysteresis loops.

These differences can be better seen by enlarging the field region in which magnetization switching events take place (Fig. 2 b bottom panels). Inspection of these curves reveals that the switching of RT-NW takes place in several steps. This is typically found when magnetization reversal follows a nucleation/propagation process, as discussed previously. Starting from the quasi-single domain saturated state at large positive (negative) magnetic fields, the first step observed in the hysteresis curve when decreasing (increasing) the sweeping magnetic field stems from the nucleation of the small reversed region and the corresponding domain wall. This serves to define the experimental nucleation fields, i.e., $H_{\mathrm{n}}^{+}$and $H_{\mathrm{n}}^{-}$, for increasing and decreasing magnetic fields, respectively (as an example, one value of $H^{+}$is indicated by an arrow in Fig. $2 \mathbf{b}$ bottom panel). The presence of steps suggests that RT-NW is not completely homogeneous, containing structural defects, impurities or a certain degree of surface roughness that behaves as pinning defects for the moving domain wall.

On the other side, magnetization reversal of the rest of the NWs takes place in extremely well defined single switching events (indicated by the respective arrows in Fig. 2b), defining the nucleation fields $H_{\mathrm{n}}^{ \pm}$. This suggests that these NWs have fewer (or a different kind of) defects as compared to RT-NW. In the case of 300-NW, an additional minor step (thick green arrows) is always observed at large (positive and negative) magnetic fields, after the main switching event has taken place. Interestingly, in the case of $600-\mathrm{NW}$, and for decreasing 
sweeping magnetic fields only, three clearly separated switching events can be distinguished. These are highlighted by three arrows (black and grey) in Fig. 2b. The latter suggests the occurrence of three different reversal paths that are undertaken stochastically.

We highlight that such peculiarities become apparent only for single shot measurements performed on individual NWs. To illustrate this, in Fig. 2c we plot the hysteresis loops resulting after averaging over $30-50$ curves measured at $15 \mathrm{~K}$. This serves to mimic the results that one would obtain when measuring a large number of identical NWs. The same would apply to measurements where averaging large number of hysteresis loops is required to achieve a tolerable signal-to-noise ratio, i.e., typical MOKE signals. For instance, a reversal process consisting of many-step events as that of RT- or 300-NW can not be distinguished from the occurrence of distinct switching events as the case of $600-\mathrm{NW}$.

Temperature dependence of $\left\langle H_{\mathrm{n}}\right\rangle$. Differences between the NWs become even more evident when performing measurements at variable temperature. The $T$-dependence of $\left\langle H_{\mathrm{n}}\right\rangle$ is shown in Fig. 3. Here, $\left\langle H_{\mathrm{n}}\right\rangle=\left(\left\langle H_{\mathrm{n}}^{+}\right\rangle-\left\langle H_{\mathrm{n}}^{-}\right\rangle\right) / 2$ with $\left\langle H_{\mathrm{n}}^{ \pm}\right\rangle$being the mean value of $H_{\mathrm{n}}^{ \pm}$ after averaging over 30-50 curves measured at each temperature. In the case of RT-NW, $\left\langle H_{\mathrm{n}}\right\rangle$ values decrease with increasing temperature roughly following a power-law. In the case of $150-\mathrm{NW},\left\langle H_{\mathrm{n}}\right\rangle$ exhibits three regimes: it increases first with increasing temperature up to $T<30 \mathrm{~K}$; for $T>30 \mathrm{~K},\left\langle H_{\mathrm{n}}\right\rangle$ decreases showing a large step at $T=60 \mathrm{~K}$. A similar step can be also observed for 300-NW. In these two later cases, i.e., 150-NW and 300-NW, $\left\langle H_{\mathrm{n}}\right\rangle$ depends almost linearly on $T$. Finally, in the case of 450- and 600-NW, $\left\langle H_{\mathrm{n}}\right\rangle$ decreases for increasing temperature and a clear flattening is observed at low temperatures below $\sim 20 \mathrm{~K}$.

Such decrease of the nucleation field as the temperature is increased is found when magnetization reversal follows a thermally activated process, as typical in magnetic nanoparticles. On the other hand, the remarkable differences between the $T$-dependence of the nucleation field of each NW (power-law, linear or T-independent behavior at low temperatures) point to a quite intricate scenario. We can speculate that these differences are due to structural changes going on in the NWs at intermediate annealing temperatures that lead to different 
nucleation mechanisms for the reversed domain. ${ }^{32}$ This issue will be treated in detail in a separated publication.

In the case of RT-NW, the large scattering found in the $\left\langle H_{\mathrm{n}}\right\rangle$ vs $T$ values suggest that this NW might find multiple almost-equivalent paths to reverse their magnetization. This can be due to the presence of pinned Co atoms or antiferromagnetic species, e.g., CoO or $\mathrm{Co}_{3} \mathrm{O}_{4}$. These oxides are very likely to be present at the surface of the NW and even in the core. Interaction between ferromagnetic Co nanocrystals and pinned Co atoms or antiferromagnetic regions leads to spin frustration that typically exhibits a non-reproducible (spin glass-like) behavior along different measurements. In this scenario, the energy landscape and, therefore, the effective reversal path, varies fairly from one hysteresis measurement to the other leading to a quite broad distribution of nucleation fields.

Finally, the behavior of $150-$ and $300-\mathrm{NW}$ can be tentatively explained as follows. The hysteresis loops of 150-NW measured at $T<35 \mathrm{~K}$ are slightly shifted towards positive $H$ values (see Fig. 2b). In this temperature range the nucleation field also exhibits other counter-intuitive behavior, like the fact that $\left\langle H_{\mathrm{n}}\right\rangle$ increases for increasing temperatures. This is contrary to what is expected for a thermally activated process and is usually found in exchange-biased systems. ${ }^{33}$ It is tempting to associate such a behavior with the presence of some amount of $\mathrm{Co}_{3} \mathrm{O}_{4}$ phase being antiferromagnetic below $\sim 35 \mathrm{~K}$. CoO has a very large Neél temperature $T_{\mathrm{N}}$ close to room temperature but $\mathrm{Co}_{3} \mathrm{O}_{4}$ exhibits $30<T_{\mathrm{N}}<40 \mathrm{~K}$, typically. ${ }^{34}$ At $T<T_{\mathrm{N}}$, the exchange bias effect might lead to the aforementioned positive $H$ shift. ${ }^{35}$ This effect disappears at $T>35 \mathrm{~K}$. In this temperature range $\left\langle H_{\mathrm{n}}\right\rangle$ values decrease for increasing temperature and hysteresis loops are well centered around zero field (not shown). In addition to that, a small negative $H$-shift is observed at $T>60 \mathrm{~K}$ for both 150 - and 300-NW. A more comprehensive analysis of this behavior will be also given in a separate publication.

Effects of the annealing temperature on the crystalline structure. Regarding the internal structure of the NWs it will be useful to focus on the experimentally measured 
nucleation field of each NW at fixed temperature, e.g., $T=1.4 \mathrm{~K}$. It is important to highlight that, according to the simple curling model of magnetization reversal, the nucleation field does not depend on $p$ but depends strongly on the radius of the NW as $\propto 1 / R^{2}$. The same also applies to more realistic models including nucleation/propagation of magnetic domains. This can be easily seen by performing micromagnetic simulations and considering the actual geometry of each NW (see Methods section). Simulations assume zero temperature and neglect any contribution of the magnetocrystalline anisotropy. In Fig. 4a we plot the numerically calculated nucleation fields multiplied by $R^{2}$ (solid line). These results must be compared with $\left\langle H_{\mathrm{n}}^{1.4}\right\rangle R^{2}$, i.e, the averaged nucleation field measured for each NW at the lowest experimental temperature $(T=1.4 \mathrm{~K})$ multiplied by $R^{2}$ (colored dots). As expected, numerical calculations lead to an approximately flat horizontal curve. Interestingly, experimental points exhibit a striking crossover: $\left\langle H_{\mathrm{n}}^{1.4}\right\rangle R^{2}$ corresponding to RT- and 150-NW lie well below the calculated data points whereas values obtained for 300-, 450- and 600-NW are much larger than expected. The behavior of RT- and 150-NW is not surprising since defects, unavoidable in real NWs, behave as localized nucleation points for reversed domains. This decreases the effective energy barriers for magnetization reversal, thus the nucleation fields. These effects can be emulated by means of numerical simulations. For this purpose, we assume that there are a number $(\sim 250)$ of randomly distributed pinning centers $(5 \mathrm{~nm}-$ radius spheres) having randomly fixed magnetization. In these simulations we also neglect the contribution of the magnetocrystalline anisotropy. The results can be seen in Fig. 4a (open stars). Interestingly, the numerically calculated nucleation fields are largely reduced as compared to those obtained in the absence of pinning centers. As discussed previously, pinned defects serve as nucleation centers for the reversed magnetization, triggering switching.

On the other hand, the behavior of the NWs annealed at larger temperatures suggests that magnetocrystalline anisotropy, $K_{\mathrm{u}}$, can not be neglected in these samples. This can be implemented in the numerical simulations by assuming a net uniaxial anisotropy constant $K_{\mathrm{u}}=\beta K_{\mathrm{u}}($ bulk $)$ where $K_{\mathrm{u}}($ bulk $)=5.2 \times 10^{5} \mathrm{~J} / \mathrm{m}^{3}$ is that of bulk crystalline Co. As 

simulations setting $\beta=0.09$ (crosses). This behavior might originate from the crystallization of larger and larger pure Co crystals as annealing temperature increases. To prove this, High Resolution Transmission Electron Microscopy (HRTEM) imaging has been performed to monitor the structural change of the NWs with increasing annealing temperature (Fig. $4 \mathbf{b}$ ). The RT-NW shows the typical nanocrystalline microstructure, with no texture, as the fast Fourier transform (FFT) of a small area of the image evidences a diffuse diffraction ring. For the 150-NW, some individual Bragg diffraction spots can be observed in combination with the diffuse diffraction ring, which indicates the presence of grains of larger size. In the 300-NW case, the diffuse scattering has vanished and areas with superposition of different crystals are most commonly detected. In the 450-NW, the FFT patterns reveal that larger areas exhibit a microstructure composed of single crystals. In this case, the grain size becomes similar to the NW diameter, identifying fewer regions with overlapping between different crystals. Finally, in the case of $600-\mathrm{NW}$, the nanostructure is constituted by several large crystals, whose lateral size is the NW width and separated from each other by grain boundaries expanded along the whole diameter. Examples of different crystal structures are shown in Fig. 4b, where face-centered-cubic $(f c c)$ and hexagonal-closest-packed (hcp) Co crystals have been found.

Effects of the annealing temperature on the Co purity. Magnetization measurements allow us extracting information about the Co purity as well. From the total signal captured by each nanoSQUID $\left(\Phi_{\text {exp }}\right.$, see Fig. 2 b) it is possible to numerically estimate the total magnetic moment of the NWs $\left(\mu_{\mathrm{NW}}\right)$. For this purpose, experimental values of $\Phi_{\text {exp }}$ must be compared with the calculated flux $\Phi_{\text {theo }}=\left|\phi_{\mu}\right| \mu_{\mathrm{NW}}$, where $\phi_{\mu}$ is the averaged coupling factor for each nanoSQUID across the NW volume (see Methods section). The obtained values of $\mu_{\mathrm{NW}}$ and the resulting Co purity, defined as $p=\mu_{\mathrm{NW}} / V_{\mathrm{mag}} M_{\mathrm{s}}(\mathrm{bulk})$, are given in Table 1. Remarkably, $p$ increases for increasing annealing temperature in very good agreement with results obtained from Electron Energy Loss Spectroscopy (EELS) chemical 
analysis in Scanning Transmission Electron Microscopy (STEM) mode, as shown in Fig. 4c. The average Co composition increases from 72 atom \% of RT-NW up to $78 \%$ for 150-NW, $88 \%$ for 300-NW, $92 \%$ for 450-NW and $94 \%$ for 600-NW. Accordingly, the core average net magnetic induction, obtained by off-axis electron holography experiments (see Fig. 4d), increases from $0.91 \mathrm{~T}$ for RT-NW up to $1.22 \mathrm{~T}$ for $150-\mathrm{NW}, 1.36 \mathrm{~T}$ for $300-\mathrm{NW}, 1.49 \mathrm{~T}$ for 450-NW and $1.61 \mathrm{~T}$ for $600-\mathrm{NW}$, reaching a value very close to the bulk magnetic induction of $1.76 \mathrm{~T}$.

\section{Discussion}

Considering these experimental data, it is possible to provide a tentative interpretation of the results. According to our observations, samples could be divided into three groups.

RT-NW. The RT-NW exhibits clear signs of high structural disorder and an important degree of magnetic frustration leading to a spin glass-like behavior. This is evidenced by the presence of a multiple-step magnetization reversal ( Fig. 2b) and the large scattering observed in the $\left\langle H_{\mathrm{n}}\right\rangle$ vs $T$ values (see Fig. 3) The $T$-dependence of $\left\langle H_{\mathrm{n}}\right\rangle$ follows the typical power law observed for thermally assisted magnetization reversal in nanoparticles.

150- and 300-NW. Annealing the NWs seems to reduce the degree of disorder as suggested by the reduced number of steps in the hysteresis loops ( Fig. 2b). In addition to that, we observe a striking trend change in the $T$-dependence of $\left\langle H_{\mathrm{n}}\right\rangle$ that becomes roughly linear ( Fig. 3) together with an important increase of the nucleation field (crossover shown in Fig. 4a). These observations point to significant structural changes taking place in the annealed NWs having an influence on the switching mechanisms and the effective anisotropy of the NWs.

On the other hand, the presence of antiferromagnetic species becomes much more evident. This is manifested by the non-symmetric hysteresis loops observed in 150-NW below $35 \mathrm{~K}$ ( Fig. 2b) and the fact that $\left\langle H_{\mathrm{n}}\right\rangle$ increases for increasing temperature (Fig. 3). The latter 
points to the existence of substantial antiferromagnetic crystals that become paramagnetic above $T \sim 35 \mathrm{~K}$. A similar effect is observed for both 150 - and $300-\mathrm{NW}$ at $T>60 \mathrm{~K}$ where hysteresis loops are slightly shifted towards negative magnetic fields. Interestingly, oxygenrich regions nucleate in the NW's core precisely for annealing temperatures between 150 and $300{ }^{\circ} \mathrm{C}$. This is evidenced by the oxygen chemical maps obtained from EELS measurements (Fig. 4c). The observed oxygen-rich regions may well correspond to relatively large $(\sim 10$ $\mathrm{nm}$ ) Co oxide nanoparticles that would lead to the well known phenomenon of exchange bias observed experimentally.

450- and 600-NW. Finally, NWs annealed at 450 and $600{ }^{\circ} \mathrm{C}$ behave very similar. Both exhibit a similar dependence of $\left\langle H_{\mathrm{n}}\right\rangle$ on $T$ (Fig. 3) and increased values of the nucleation fields (Fig. 4a) suggesting an effectively increased (magnetocrystalline) anisotropy as discussed previously. Remarkably, HRTEM images and FFT patterns provide the existence of both $h c p$ and $f c c$ Co crystals, forming quite large $(\sim 100 \mathrm{~nm})$ crystalline regions separated by grain boundaries ( Fig. 4c). Being randomly distributed, such crystalline grains might lead to a net uniaxial magnetocrystalline anisotropy that would indeed increase the resulting nucleation fields. On the other hand, the presence of antiferromagnetic species or spin frustration is still suggested by the observation of multiple co-existing reversal paths ( Fig. 2b). Indeed, oxygen-rich regions are still visible in the STEM-EELS chemical map shown in Fig. 4c for 450-NW.

\section{Conclusions}

In summary, we have presented a detailed study of the magnetic properties of individual Co-FEBID NWs annealed at different temperatures. This has been possible thanks to the enormous spin sensitivity and broad field and temperature operation range of YBCO nanoSQUID sensors. Experimental results point clearly to an enhanced Co content and increased degree of crystallinity in NWs annealed at large temperatures $\left(450\right.$ and $\left.600{ }^{\circ} \mathrm{C}\right)$. 
The latter results in an increased effective magnetocrystalline anisotropy, as revealed by the exceedingly large values for the switching magnetic fields. On the other hand, antiferromagnetic species have a limited influence in these NWs. These observations agree very well with the structural and chemical characterization performed on NWs grown and annealed under the same experimental conditions. ${ }^{27}$ Ex-situ annealing magnetic FEBID nanostructures seems, therefore, to be a very promising strategy to routinely produce high-quality, pure and crystalline nanomagnets with large spatial and lateral resolution. In addition, our measurements demonstrate the enormous convenience of using YBCO nanoSQUID sensors for the magnetic characterization of nanoscopic magnets.

\section{Methods}

\section{Fabrication of Co-FEBID NWs}

The working principle of FEBID is similar to chemical vapour deposition assisted by an electron beam. Co-FEBID NWs are fabricated using $\mathrm{Co}_{2}(\mathrm{CO})_{8}$ precursor gas in an FEI Helios Nanolab 650 dual-beam system. The electron beam current is kept to 100 pA leading to total deposition times of 40 - 60 s. RT-NW is fabricated onto a $500 \mathrm{~nm}$-thick $\mathrm{Si}_{3} \mathrm{~N}_{4}$ membrane covered by $10 \mathrm{~nm}$ of $\mathrm{Al}$ avoiding charge effects (see Fig. 1a). The rest of the NWs are grown on TEM copper grids. The use of metallic grids is paramount to guarantee good thermalization of the NWs during the postgrowth ex-situ annealing treatment. These grids are previously thinned and partially cut into microscopic lamellae which facilitates the subsequent transport steps. Annealing is carried out during 100 minutes in an FEI Quanta FEG-250 scanning electron microscope equipped with a heating stage (heating ramp of $\left.50^{\circ} \mathrm{C} / \mathrm{min}\right)$. After annealing, the heater is turned off and the sample is left to cool down. 


\section{Nano-SQUID sensors}

NanoSQUIDs are made out of a 80 nm-thick YBCO film epitaxially grown on a $\mathrm{SrTiO}_{3}$ (STO) bicrystal substrate $\left(24^{\circ}\right.$ missorientation angle) and covered by $70 \mathrm{~nm}$ of gold. The STO grain boundary (GB) is naturally transferred to the YBCO film and the GB in YBCO exhibits Josephson-like behavior. Due to oxygen outdiffusion from the narrow junction and the ensuing degradation, the fabrication of submicron junctions from grain boundaries in cuprates is a difficult task. We have overcome this problem through a careful FIB milling method that produces a certain amount of redeposited amorphous YBCO and STO covering the junction edges and preventing oxygen outdiffusion. Following this method, a central loop with typical dimensions $550 \times 400 \mathrm{~nm}^{2}$ is FIB patterned, intersecting the GB (indicated as a dashed yellow line in Fig. 1b). This leads to minute inductances below a few tens of $\mathrm{pH}$ and exceptionally low values of the flux noise $\sim 1 \mu \Phi_{0} / \sqrt{\mathrm{Hz}}$ at $100 \mathrm{kHz}$. The resulting devices have non-hysteretic current-voltage-characteristics with typical critical currents $I_{c} \sim$ $500-800 \mu \mathrm{A}$ (at $4.2 \mathrm{~K}$ ) and normal state resistances $R \sim 1 \Omega$. The SQUIDs are currentbiased and operated in Flux Locked Loop (FLL) mode using commercial SQUID readout electronics. FLL operation is possible thanks to the patterning of a narrow constriction (width $\sim 180 \mathrm{~nm}$, typically) that allows coupling a net magnetic flux to the nanoloop (see Fig. 1b). The mutual inductance between the nanoloop and the constriction is $M \sim \Phi_{0} / \mathrm{mA}$.

\section{Magnetization measurements with nanoSQUID}

Each nanoSQUID containing one individual NW is mounted in good thermal contact to a sapphire plate installed in a variable temperature insert (minimum temperature $\sim 1.4 \mathrm{~K}$ ). This sapphire plate is mounted on a rotator that allows aligning the nanoSQUID with respect to the externally applied magnetic field with a resolution better than $0.1^{\circ}$. Magnetization measurements are performed by sweeping the external magnetic field at a fixed rate of $\nu=8.3 \mathrm{mT} / \mathrm{s}$ and measuring the output nanoSQUID voltage $\left(V_{\text {out }}\right)$ as the NW is driven through magnetization reversal. A total number of $30-50$ hysteresis loops are measured 
at each temperature. $V_{\text {out }}$ is converted into units of magnetic flux threading the nanoloop $\Phi_{\exp }=V_{\text {out }} M / R_{\mathrm{f}}$, where $R_{\mathrm{f}}$ is the feedback resistance of the SQUID readout electronics $\left(R_{\mathrm{f}}=3.3 \mathrm{k} \Omega\right.$, typically). Differences between the total captured flux $\left(\Phi_{\text {exp }}\right)$ from each NW are due to slight geometrical differences between the nanoSQUIDs, differences between the NWs' positions and differences in the total magnetic moment of the NWs.

The reproducibility of the fabrication procedure has been checked on 300-NW. For this purpose, a different NW grown and annealed under the same experimental conditions was deposited on a different nanoSQUID. Measurements yield very similar results to those shown here (see Fig. 2 in the Supporting Information).

\section{Calculation of the coupling factor}

In order to estimate the average coupling factor, it is necessary to know the exact position of the NW, its precise dimensions and those of the nanoSQUID. Numerical calculations are performed using the finite-element based software package 3D-MLSI: ${ }^{36}$ A circulating current is sent around the SQUID loop in 11 current sheets, ${ }^{37,38}$ taking into account the precise thickness and in-plane geometry of the device. The magnetic field generated inside the volume occupied by the NW is then used to calculate $\phi_{\mu} \cdot{ }^{23,36}$ To estimate the error resulting from the SEM-based measurements of the NW position, $\phi_{\mu}$ was recalculated with the NW position shifted by $\pm 100 \mathrm{~nm}$, using the maximum deviations as errors for $\phi_{\mu}\left(\Delta \phi_{\mu}\right)$. The calculated Co purity error given in Table 1 also includes the uncertainty in the measurement of the NW radius $(\Delta r= \pm 2 \mathrm{~nm})$ and in the experimental total signal coupled to the $\operatorname{nanoSQUID}\left(\Delta \Phi_{\exp }= \pm 10 \mathrm{~m} \Phi\right)$.

\section{Microstructural, compositional and magnetic analyses by TEM}

HRTEM imaging was carried out in a Titan Cube 60-300 system operated at $300 \mathrm{kV}$, equipped with an S-FEG, a CETCOR aberration corrector for a objective lens from CEOS providing subangstrom point resolution, and a $2 K \times 2 K$ Ultrascan CCD camera from Gatan. 
STEM and EELS experiments were performed in a Titan Low-Base 60-300 system operated at $300 \mathrm{kV}$, fitted with a high-brightness field emission gun (X-FEG) and a CETCOR corrector for the condenser system, which produces an electron probe with a lateral size below $1 \AA$. The STEM-EELS experiments were performed using a Gatan Image Filter (GIF) Tridiem 866 ERS, with a 25 mrad convergence semiangle, an energy dispersion of $0.5 \mathrm{eV} /$ pixel with a resolution of $1.5 \mathrm{eV}$, a GIF aperture of $2.5 \mathrm{~mm}$, a camera length of $10 \mathrm{~mm}$, a pixel time of $15 \mathrm{~ms}$, and an estimated beam current of $270 \mathrm{pA}$. The magnetic induction was determined by off-axis Electron Holography in the Titan Cube 60-300 system mentioned above. These holographic experiments were performed at $300 \mathrm{kV}$ by switching off the objective lens and using the Lorentz lens to form the image. The detailed experimental procedure can be found elsewhere. ${ }^{28}$ A motorized electrostatic biprism was excited at $150-170 \mathrm{~V}$ to generate an interferometric pattern in an overlapping area of 400-500 $\mathrm{nm}$ in width, with a fringe contrast ranging from $20 \%$ to $25 \%$. The hologram acquisition time was $5 \mathrm{~s}$, and they were acquired at remanence state after the saturation of the magnetization in the two opposite directions along the longitudinal NW axis by tilting the object by $30^{\circ}$ and exciting the objective lens up to a magnetic field of $0.3 \mathrm{~T}$. Thus, the electrostatic phase shift can be subtracted and the magnetic phase shift $\left(\varphi_{M A G}\right)$ extracted. If the NW axis is set along the $x$ axis, the magnetic induction component $B_{x}$ can be obtained as $\left|B_{x}(x, y)\right|=(\hbar / e t)\left[\partial \varphi_{M A G}(x, y) / \partial y\right]$, where $\hbar$ is the reduced Planck constant, $e$ the electron charge, and $t$ the total variable thickness along the NW width. ${ }^{28}$

\section{Micromagnetic simulations}

Micromagnetic simulations are performed using the GPU-accelerated MUMAX ${ }^{3}$ package. ${ }^{39}$ We set the saturation magnetization $M_{\mathrm{s}}=p \times M_{\mathrm{s}}(\mathrm{bulk})$ and the exchange stiffness $A=$ $p \times A\left(\right.$ bulk) with $p$ given in Table 1 and $M_{\mathrm{s}}($ bulk $)=1.4 \times 10^{6} \mathrm{~A} / \mathrm{m}$ and $A($ bulk $)=2.5 \times 10^{-11}$ $\mathrm{J} / \mathrm{m}$ for pure crystalline Co. On the other hand, the size of each NW is set to its actual geometrical dimensions given in Table 1 . The cell size is set to $2.4 \times 2.4 \times 3.5 \mathrm{~nm}^{3}$ which is 
below the exchange length of bulk Co $\left(\lambda_{\mathrm{ex}} \sim 4.5 \mathrm{~nm}\right)$.

\section{Supporting information}

NW transport details and procedure reproducibility.

\section{Acknowledgments}

This work was partly funded and supported by the Spanish MINECO (MAT2014-51982C2, MAT2015-73914-JIN, MAT2015-64083-R, MAT2015-69725-REDT, MAT2017-82970-C21-R and MAT2017-82970-C2-2-R), the Aragón Regional Government through projects E26, E09_17R and E13_17R (Construyendo Europa desde Aragón), COST Action CA16218, COST Project CELINA and the Deutsche Forschungsgemeinschaft via project KO 1301/132. J.P.-N. grant is funded by the Ayuda para Contratos Predoctorales para la Formación de Doctores of the spanish MINECO (BOE 12/06/15) with the participation of the European Social Fund. B. M. gratefully acknowledges support by the German Academic Scholarship Foundation. The Research Support Services and the staff at the Advanced Microscopy Laboratory from the University of Zaragoza are acknowledged.

\section{References}

(1) Fernández-Pacheco, A.; Streubel, R.; Fruchart, O.; Hertel, R.; Fischer, P.; l P. Cowburn, R. Three-dimensional nanomagnetism. Nat. Commun. 2017, ㅁ, 15756.

(2) Parkin, S. S. P.; Hayashi, M.; Thomas, L. Magnetic Domain-Wall Racetrack Memory. Science 2008, 320, 190-194.

(3) Allwood, D. A.; Xiong, G.; Faulkner, C. C.; Atkinson, D.; Petit, D.; Cowburn, R. P. Magnetic Domain-Wall Logic. Science 2005, 309, 1688-1692. 
(4) Poggio, M.; Degen, C. L. Force-detected nuclear magnetic resonance: recent advances and future challenges. Nanotechnology 2010, 21, 342001.

(5) Vavassori, P.; Pancaldi, M.; Perez-Roldan, M. J.; Chuvilin, A.; Berger, A. Remote Magnetomechanical Nanoactuation. Small 2016, 12, 1013-1023.

(6) Col, S. D.; Jamet, S.; Rougemaille, N.; Locatelli, A.; Mentes, T. O.; Burgos, B. S.; Afid, R.; Darques, M.; Cagnon, L.; Toussaint, J. C.; Fruchart, O. Observation of Blochpoint domain walls in cylindrical magnetic nanowires. Phys. Rev. B 2014, $\underline{89}$.

(7) Vazquez, M., Ed. Magnetic nano- and microwires: design, synthesis, properties and applications; Woodhead Publishing, 2015.

(8) Fernández-Pacheco, A.; Serrano-Ramón, L.; Michalik, J. M.; Ibarra, M. R.; De Teresa, J. M.; O’Brien, L.; Petit, D.; Lee, J.; Cowburn, R. P. Three dimensional magnetic nanowires grown by focused electron-beam induced deposition. Sci. Rep. 2013, $\underline{3}, 1492$.

(9) Keller, L.; Mamoori, M. K. I. A.; Pieper, J.; Gspan, C.; Stockem, I.; Schröder, C.; Barth, S.; Winkler, R.; Plank, H.; Pohlit, M.; Müller, J.; Huth, M. Direct-write of free-form building blocks for artificial magnetic 3D lattices. Sci. Rep. 2018, $\underline{8}$.

(10) Lipert, K.; Bahr, S.; Wolny, F.; Atkinson, P.; Weißker, U.; Mühl, T.; Schmidt, O. G.; Büchner, B.; Klingeler, R. An individual iron nanowire-filled carbon nanotube probed by micro-Hall magnetometry. Appl. Phys. Lett. 2010, 97, 212503.

(11) Pablo-Navarro, J.; Sanz-Hernández, D.; Magén, C.; Fernández-Pacheco, A.; de Teresa, J. M. Tuning shape, composition and magnetization of 3D cobalt nanowires grown by focused electron beam induced deposition (FEBID). J. Phys. D 2017, $\underline{50,}$ $18 \mathrm{LT} 01$. 
(12) Rodríguez, L.; Magén, C.; Snoeck, E.; Gatel, C.; Marín, L.; Serrano-Ramón, L.; Prieto, J.; Muñoz, M.; Algarabel, P.; Morellon, L.; Teresa, J. D.; Ibarra, M. Quantitative in situ magnetization reversal studies in Lorentz microscopy and electron holography. Ultramicroscopy 2013, 134, 144-154.

(13) Wolf, D.; Rodriguez, L. A.; Béché, A.; Javon, E.; Serrano, L.; Magen, C.; Gatel, C.; Lubk, A.; Lichte, H.; Bals, S.; Tendeloo, G. V.; Fernández-Pacheco, A.; Teresa, J. M. D.; Snoeck, E. 3D Magnetic Induction Maps of Nanoscale Materials Revealed by Electron Holographic Tomography. Chem. Mater. 2015, 27, 6771-6778.

(14) Buchter, A. et al. Reversal Mechanism of an Individual Ni Nanotube Simultaneously Studied by Torque and SQUID Magnetometry. Phys. Rev. Lett. 2013, 111, 067202.

(15) Ganzhorn, M.; Klyatskaya, S.; Ruben, M.; Wernsdorfer, W. Carbon Nanotube Nanoelectromechanical Systems as Magnetometers for Single-Molecule Magnets. ACS Nano 2013, $\underline{7}, 6225-6236$.

(16) Granata, C.; Vettoliere, A. Nano Superconducting Quantum Interference device: A powerful tool for nanoscale investigations. Phys. Rep. 2016, $\underline{614}$, 1-69.

(17) Martínez-Pérez, M. J.; Koelle, D. NanoSQUIDs: Basics \& recent advances. Phys. Sci. Rev. 2017, 2, 20175001.

(18) Martínez-Pérez, M. J.; Gella, D.; Müller, B.; Morosh, V.; Wölbing, R.; Sesé, J.; Kieler, O.; Kleiner, R.; Koelle, D. Three-Axis Vector Nano Superconducting Quantum Interference Device. ACS Nano 2016, 10, 8308-8315.

(19) Vasyukov, D.; Ceccarelli, L.; Wyss, M.; Gross, B.; Schwarb, A.; Mehlin, A.; Rossi, N.; Tütüncüoglu, G.; Heimbach, F.; Zamani, R. R.; Kovács, A.; i Morral, A. F.; Grundler, D.; Poggio, M. Imaging Stray Magnetic Field of Individual Ferromagnetic Nanotubes. Nano Lett. 2018, 18, 964-970. 
(20) Wernsdorfer, W.; Hasselbach, K.; Mailly, D.; Barbara, B.; Benoit, A.; Thomas, L.; Suran, G. DC-SQUID magnetization measurements of single magnetic particles. J. Magn. Magn. Mater. 1995, 145, 33-39.

(21) Wernsdorfer, W.; Hasselbach, K.; Benoit, A.; Barbara, B.; Doudin, B.; Meier, J.; Ansermet, J.-P.; Mailly, D. Measurements of magnetization switching in individual nickel nanowires. Phys. Rev. B 1997, 55, 11552-11559.

(22) Schwarz, T.; Nagel, J.; Wölbing, R.; Kemmler, M.; Kleiner, R.; Koelle, D. LowNoise Nano Superconducting Quantum Interference Device Operating in Tesla Magnetic Fields. ACS Nano 2013, 7, 844-850.

(23) Schwarz, T.; Wölbing, R.; Reiche, C.; Müller, B.; Martínez-Pérez, M. J.; Mühl, T.; Büchner, B.; Kleiner, R.; Koelle, D. Low-NoiseYBa2Cu3O7Nano-SQUIDs for Performing Magnetization-Reversal Measurements on Magnetic Nanoparticles. Phys. Rev. Appl. 2015, 3, 044011.

(24) Martínez-Pérez, M. J.; Müller, B.; Schwebius, D.; Korinski, D.; Kleiner, R.; Sesé, J.; Koelle, D. NanoSQUID magnetometry of individual cobalt nanoparticles grown by focused electron beam induced deposition. Supercond. Sci. Technol. 2017, 30, 024003.

(25) Vivas, L. G.; Escrig, J.; Trabada, D. G.; Badini-Confalonieri, G. A.; Vázquez, M. Magnetic anisotropy in ordered textured Co nanowires. Appl. Phys. Lett. 2012, 100, 252405 .

(26) De Teresa, J. M.; Fernández-Pacheco, A.; Córdoba, R.; Serrano-Ramón, L.; Sangiao, S.; Ibarra, M. R. Review of Magnetic Nanostructures Grown byFocused Electron Beam Induced Deposition (FEBID). J. Phys. D: Appl. Phys. 2016, 49, 243003.

(27) Pablo-Navarro, J.; Magén, C.; de Teresa, J. M. Purified and Crystalline ThreeDimensional Electron-Beam-Induced Deposits: The Successful Case of Cobalt for High Performance Magnetic Nanowires. ACS Appl. Nano Mater. 2018, 1, 38 - 46. 
(28) Pablo-Navarro, J.; Magén, C.; de Teresa, J. M. Three-dimensional core - shell ferromagnetic nanowires grown by focused electron beam induced deposition. Nanotechnology 2016, 27, 285302 .

(29) Stoner, E. C.; Wohlfarth, E. P. A Mechanism of Magnetic Hysteresis in Heterogeneous Alloys. Philos. Trans. Royal Soc. A 1948, 240, 599-642.

(30) Hubert, A.; Schafer, R. Magnetic Domains; Springer, Berlin, 1998.

(31) Paulus, P.; Luis, F.; Kröll, M.; Schmid, G.; de Jongh, L. Low-temperature study of the magnetization reversal and magnetic anisotropy of $\mathrm{Fe}, \mathrm{Ni}$, and Co nanowires. J. Magn. Magn. Mater. 2001, 224, 180-196.

(32) Stankiewicz, J.; Luis, F.; Camón, A.; Kröll, M.; Bartolomé, J.; Blau, W. Magnetization switching of Fe nanowires at very low temperatures. J. Magn. Magn. Mater. 2004, $\underline{272-276}, 1637-1639$.

(33) Nogués, J.; Sort, J.; Langlais, V.; Skumryev, V.; Suriñach, S.; Muñoz, J.; Baró, M. Exchange bias in nanostructures. Phys. Rep. 2005, 422, 65-117.

(34) He, L.; Chen, C.; Wang, N.; Zhou, W.; Guo, L. Finite size effect on Néel temperature with $\mathrm{Co}_{3} \mathrm{O}_{4}$ nanoparticles. J. App. Phys. 2007, 102, 103911.

(35) Tripathy, D.; Adeyeye, A. O.; Chakrabarti, K.; Singh, N. Tuning the exchange bias in large area Co/CoO nanowire arrays. J. Appl. Phys. 2010, 107, $09 \mathrm{D} 705$.

(36) Khapaev, M. M.; Kupriyanov, M. Y.; Goldobin, E.; Siegel, M. Current Distribution Simulation for Superconducting Multi-Layered Structures. Supercond. Sci. Technol. 2003, 16, 24-27.

(37) Nagel, J.; Konovalenko, K. B.; Kemmler, M.; Turad, M.; Werner, R.; Kleisz, E.; Menzel, S.; Klingeler, R.; Büchner, B.; Kleiner, R.; Koelle, D. Resistively shunted 
YBa2Cu3O7grain boundary junctions and low-noise SQUIDs patterned by a focused ion beam down to $80 \mathrm{~nm}$ linewidth. Supercond. Sci. Technol. 2010, 24, 015015.

(38) Wölbing, R.; Schwarz, T.; Müller, B.; Nagel, J.; Kemmler, M.; Kleiner, R.; Koelle, D. Optimizing the spin sensitivity of grain boundary junction nanoSQUIDs - towards detection of small spin systems with single-spin resolution. Supercond. Sci. Technol. 2014, $\underline{27,} 125007$.

(39) Vansteenkiste, A.; Leliaert, J.; Dvornik, M.; Helsen, M.; García-Sánchez, F.; Waeyenberge, B. V. The design and verification of MuMax3. AIP Adv. 2014, 4, 107133.

Table 1: Geometrical parameters of each NW: radius $(R)$ excluding the non-magnetic external layer of $\sim 5 \mathrm{~nm}$, length $(l)$, magnetic volume $\left(V_{\mathrm{mag}}\right), z$ positions of the NW end tip with respect to the nanoSQUID surface, total magnetic moment $\left(\mu_{\mathrm{NW}}\right)$ and Co purity $(p)$.

\begin{tabular}{l|cccccc}
\hline & $\begin{array}{c}R \\
(\mathrm{~nm})\end{array}$ & $\begin{array}{c}l \\
(\mu \mathrm{m})\end{array}$ & $\begin{array}{c}V_{\mathrm{mag}} \\
\left(10^{6} \mathrm{~nm}^{3}\right)\end{array}$ & $\begin{array}{c}z \\
(\mathrm{~nm})\end{array}$ & $\begin{array}{c}\mu_{\mathrm{NW}} \\
\left(10^{9} \mu_{\mathrm{B}}\right)\end{array}$ & $p$ \\
\hline RT-NW & 35.5 & 1.8 & 7.13 & 379 & 1.1 & $0.75 \pm 0.26$ \\
$150-\mathrm{NW}$ & 31.5 & 2.3 & 7.17 & 1050 & 1.0 & $0.84 \pm 0.26$ \\
$300-\mathrm{NW}$ & 36.5 & 2.2 & 9.21 & 1055 & 1.4 & $0.96 \pm 0.25$ \\
450-NW & 38.5 & 1.9 & 8.85 & 980 & 1.5 & $1.06 \pm 0.30$ \\
$600-\mathrm{NW}$ & 38.0 & 2.1 & 9.53 & 483 & 1.8 & $1.06 \pm 0.26$ \\
\hline
\end{tabular}




\section{Graphical TOC Entry}

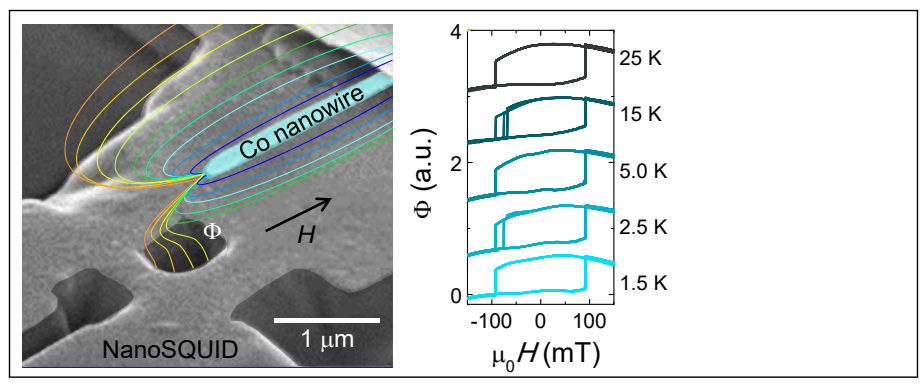



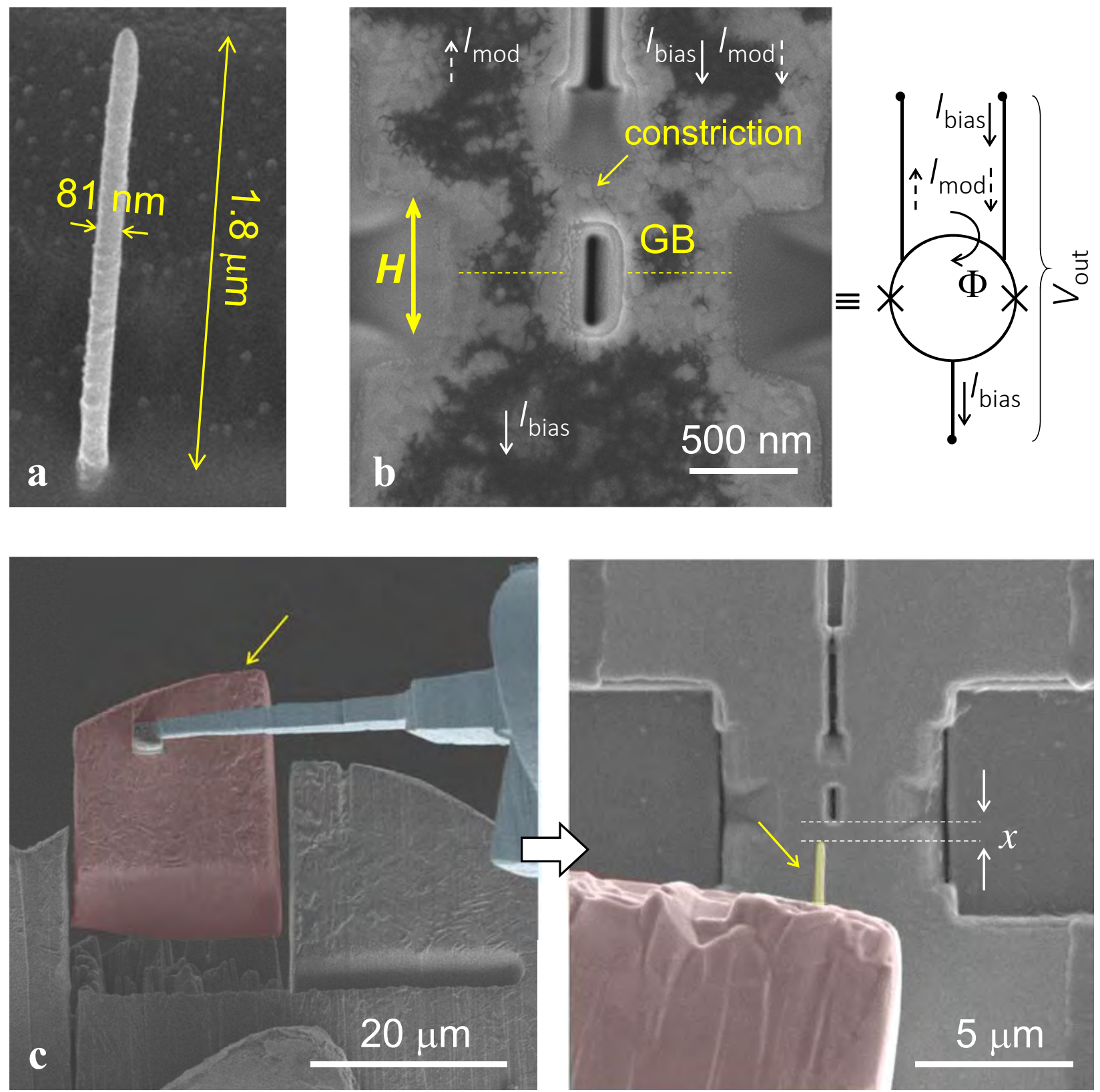

Figure 1: a: SEM image of an as-grown Co-FEBID NW. b: Typical nanoSQUID device and schematic of the equivalent electric circuit. The field $(H)$ direction, the position of the grain boundary (GB) and the constriction (see Methods for more details on the constriction) are highlighted. A current $\left(I_{\text {mod }}\right)$ flowing through the constriction couples flux $(\Phi)$ to the nanoloop serving to modulate the nanoSQUID response. $I_{\text {bias }}$ and $V_{\text {out }}$ are the biasing current and output voltage, respectively. c: False colored images of the NW transport process. The micromanipulator (blue), the copper lamella (red) and the NW (yellow arrow) are highlighted. $x$ is defined as the in-plane distance between the NW's end tip and the nanoloop edge. 

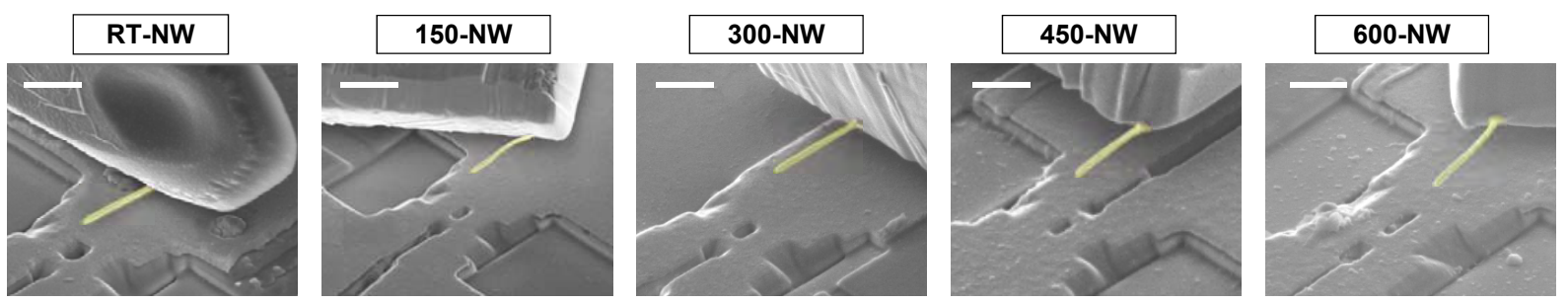

a
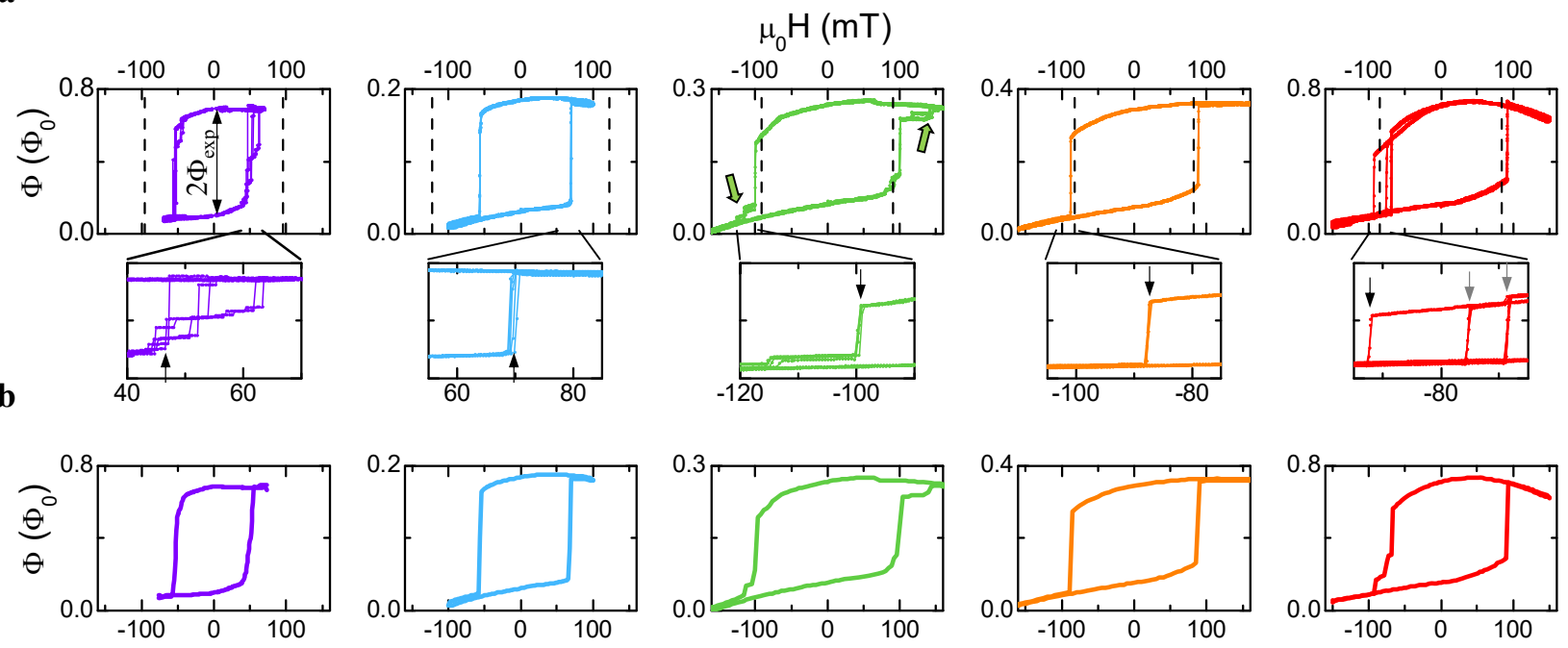

c
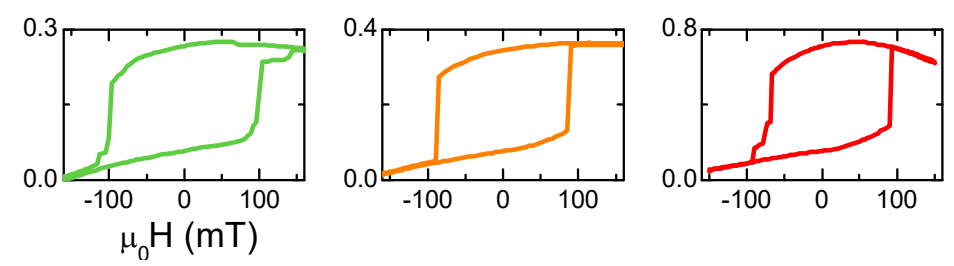

Figure 2: a: False colored SEM images of the different YBCO nanoSQUIDs with their corresponding Co-FEBID NW (yellow). Scale bars are $1 \mu \mathrm{m}$. b: Five representative hysteresis loops obtained at $15 \mathrm{~K}$ for each NW. $\Phi_{\exp }$ is the total signal coupled to the nanoSQUID from the NW. Dashed lines correspond to the theoretical nucleation fields resulting from the curling model of magnetization reversal. Bottom panels show an enlarged view of the region where magnetization switches direction. The nucleation fields $H_{\mathrm{n}}^{ \pm}$are indicated by black arrows. Gray arrows highlight the existence of distinct switching events in 600-NW whereas green thick arrows indicate additional minor steps after the main switching event in 300-NW. c: Mean hysteresis loops at $15 \mathrm{~K}$ for each NW obtained after averaging over 30-50 curves. 


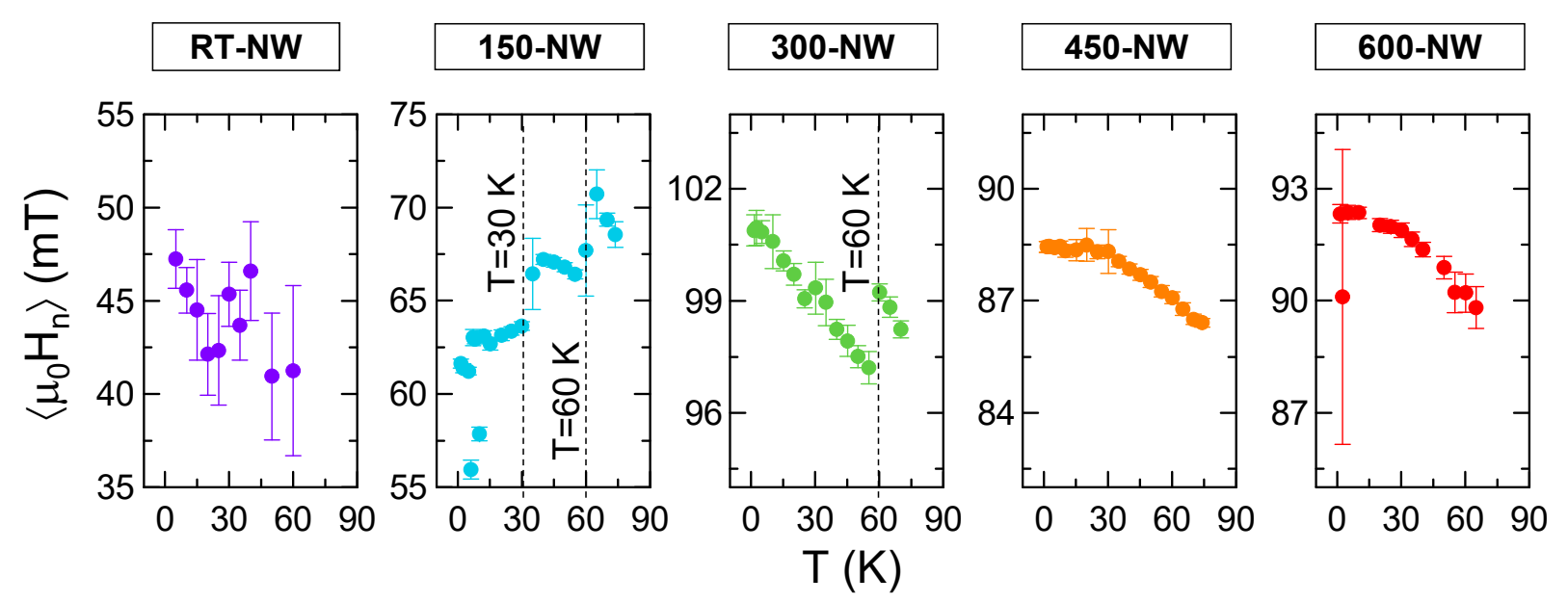

Figure 3: Temperature dependence of the averaged nucleation field for each NW. Notice that the vertical axis covers $20 \mathrm{mT}$ in the case of RT- and 150-NW, and just $10 \mathrm{mT}$ for 300-, 450and $600-\mathrm{NW}$. In the case of $150-$ and $300-\mathrm{NW}$ the relevant temperature ranges discussed in the text are highlighted. 

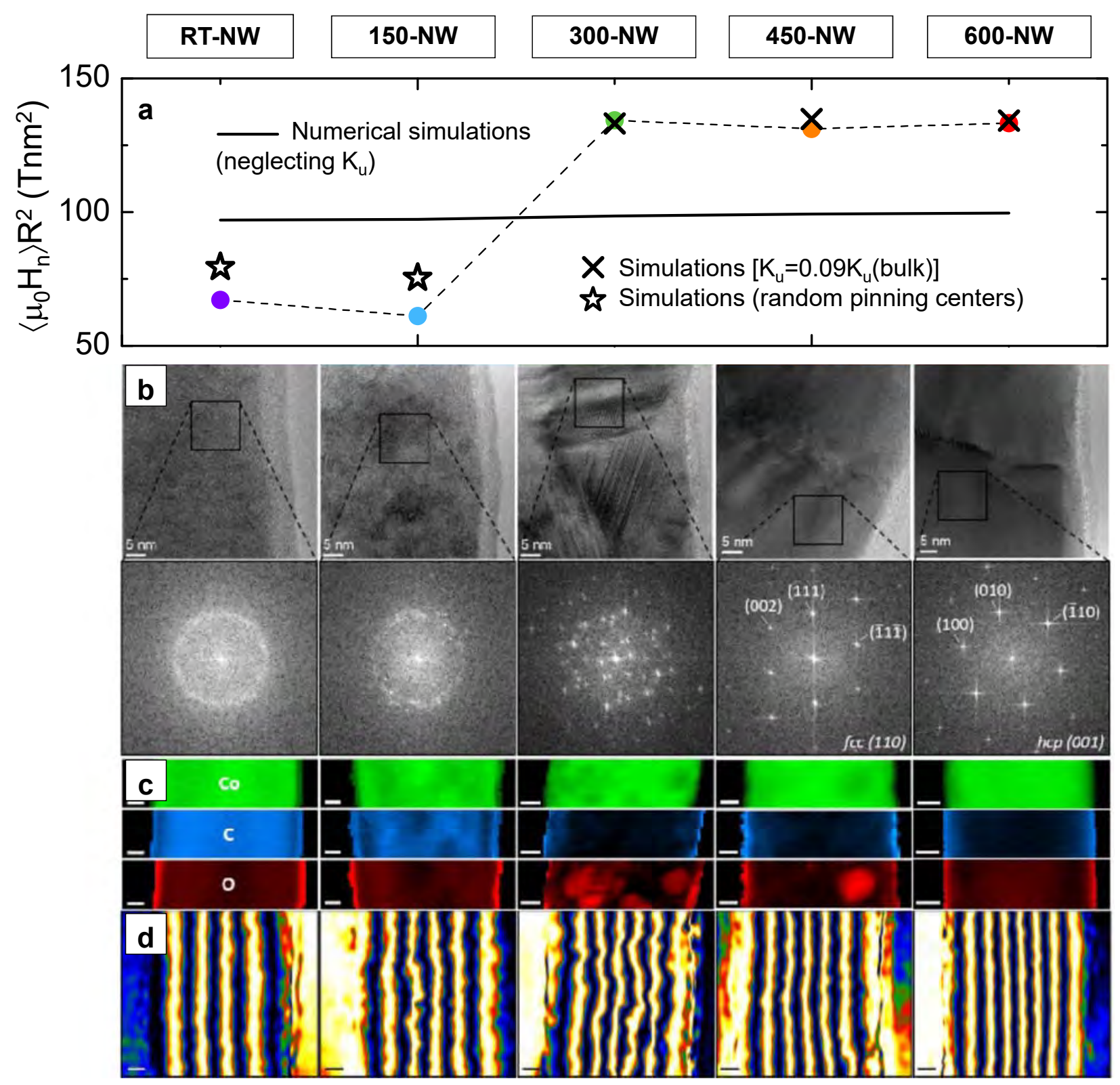

Figure 4: a: Experimentally determined (colored dots) and numerically simulated (solid line) nucleation fields at $T=0 \mathrm{~K}$ multiplied by $R^{2}$ for the NWs annealed at different temperatures. b: HRTEM images of each NW case accompanied by the corresponding FFT of a small area of the image. c: STEM-EELS chemical maps showing the spatial distribution of $\mathrm{Co}, \mathrm{C}$ and $\mathrm{O}$ in green, blue and red, respectively. d: Magnetic induction flux representations obtained by normalizing the magnetic phase images to the diameter (maximum thickness) and calculating the cosine of 350 times the normalized magnetic phase. The undefined scale bars in all images correspond to $10 \mathrm{~nm}$. Measurements shown in panels $\mathbf{b}, \mathbf{c}$ and $\mathbf{d}$ were performed on NWs grown and annealed under the same experimental conditions as those used for the nanoSQUID experiments. ${ }^{27}$ 


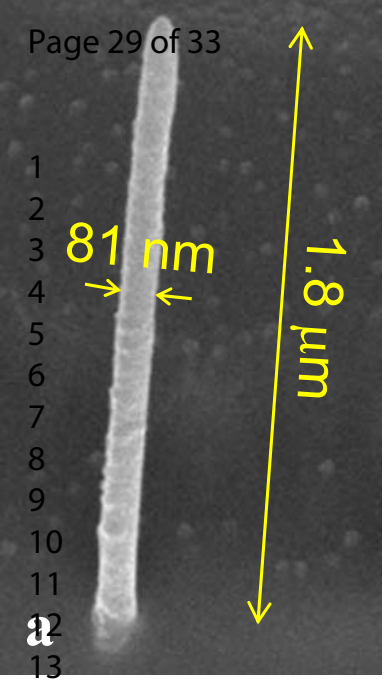

${ }_{i} /$ miturno Letters $I_{\text {bias }} I_{\text {modi }}$

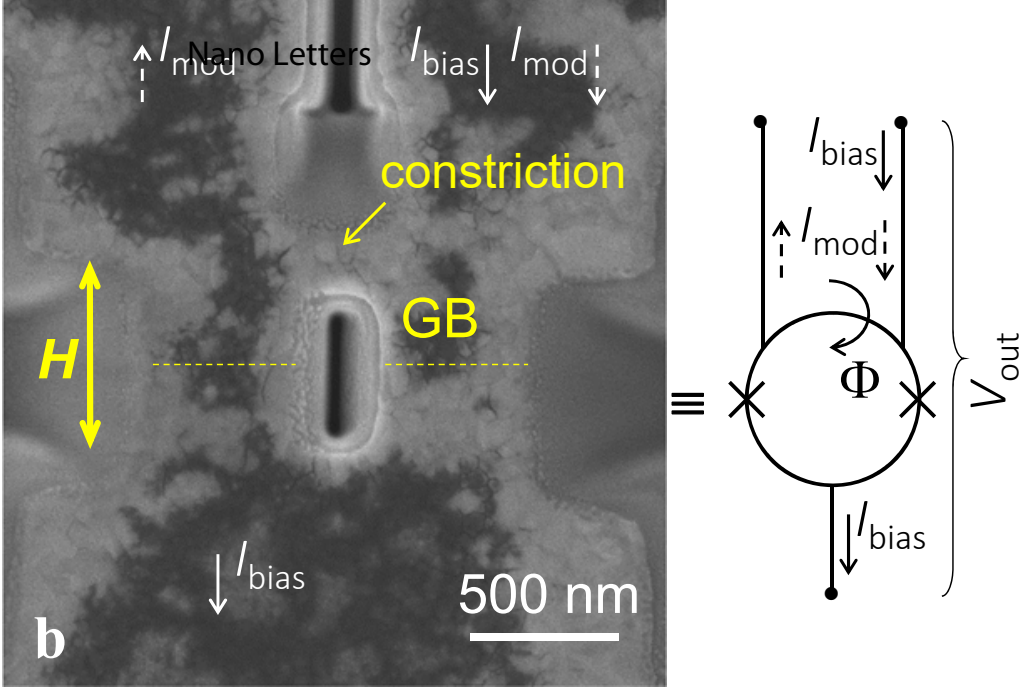

14

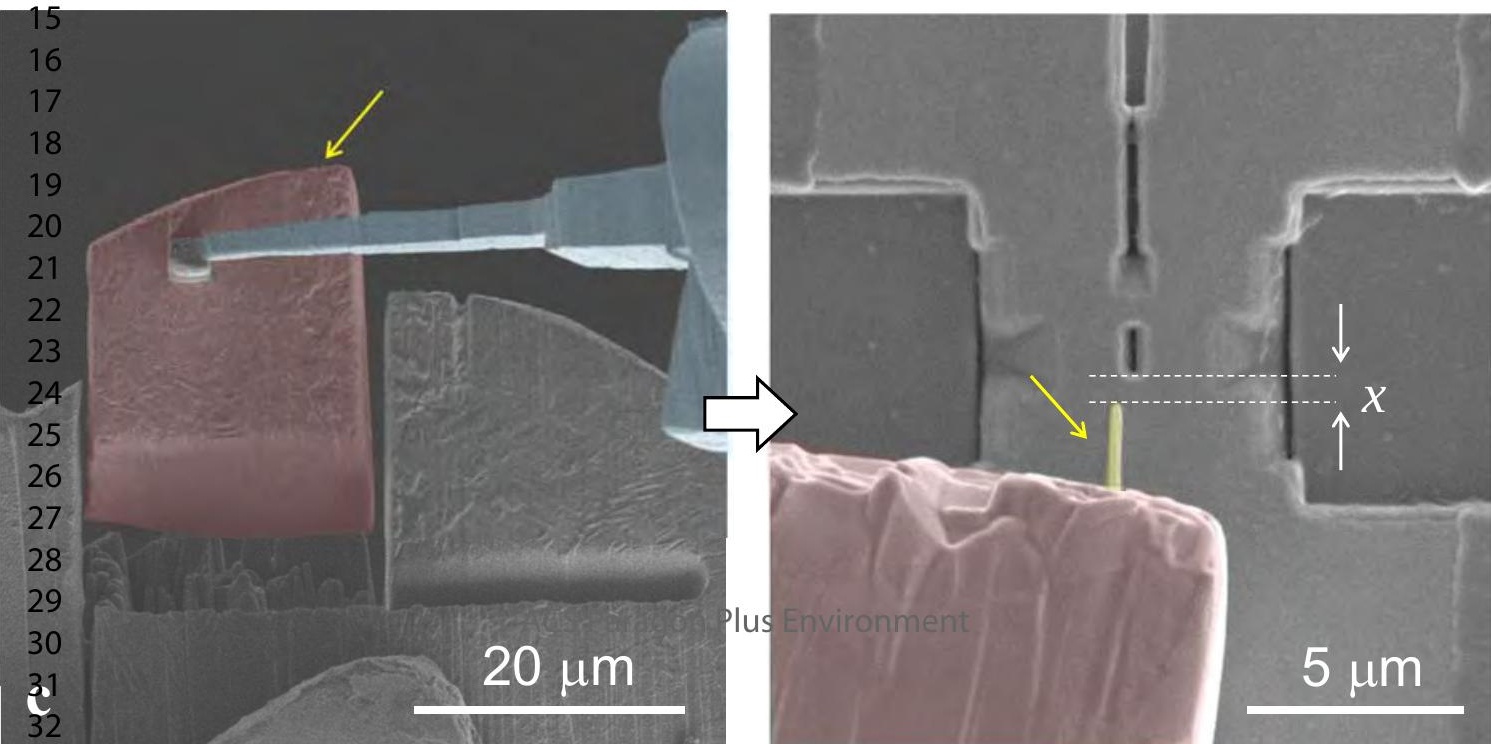




\section{RT-NW}

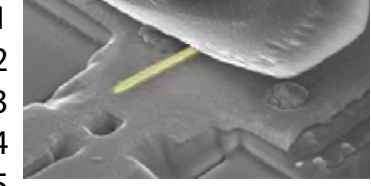
$\mathbf{a}_{6}^{5}$

7

$80.8 r_{-100,0}^{-100}$

80
P0
12
13

1

0

15

16

b 17

18

19

26

2

22

23

24

$c^{25}$

\section{0-NW}

Page 30 of 33
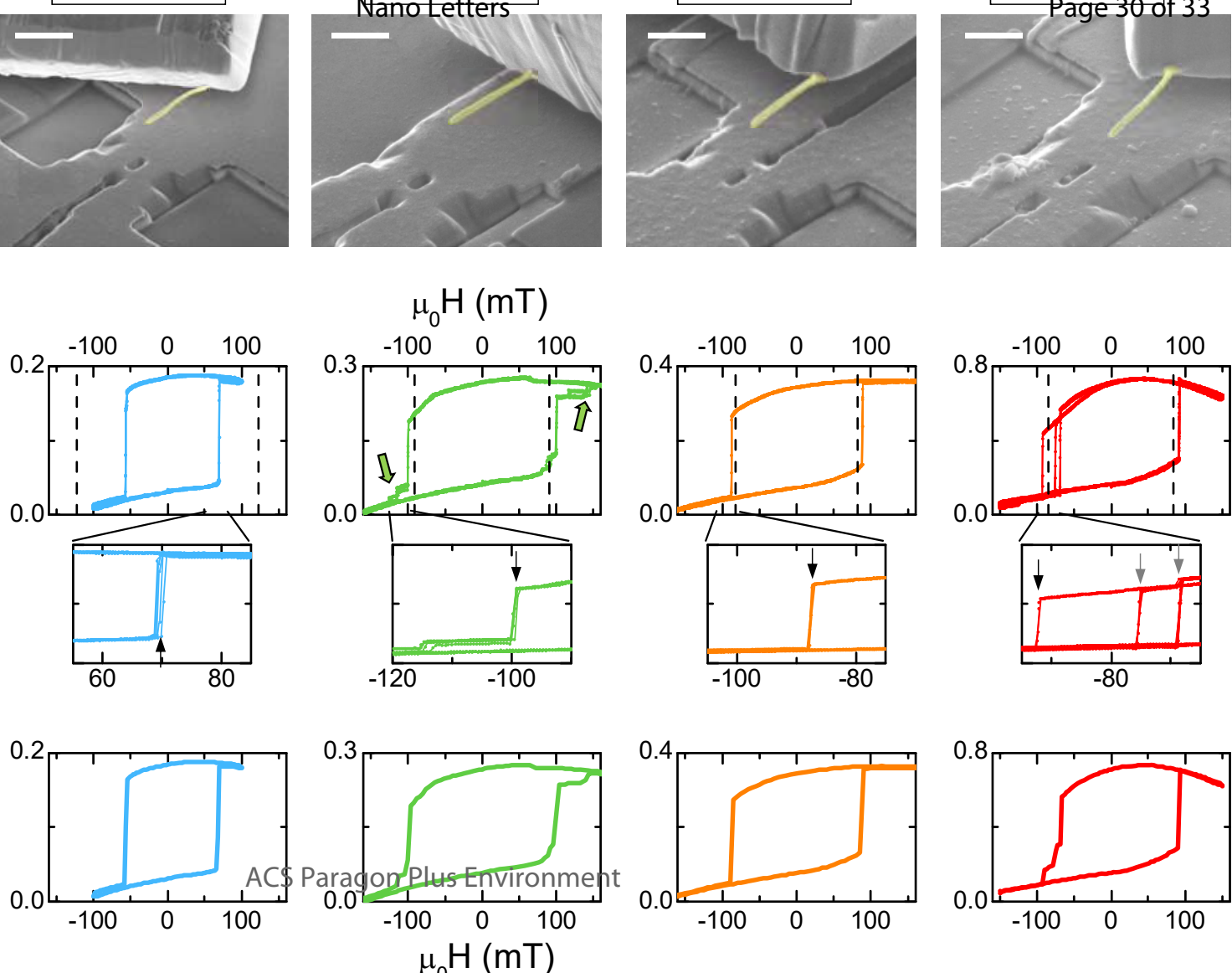
Page 31 of $\mathbf{3}$ TT-NW

\section{0-NW}

Nano BeqeNSW

450-NW

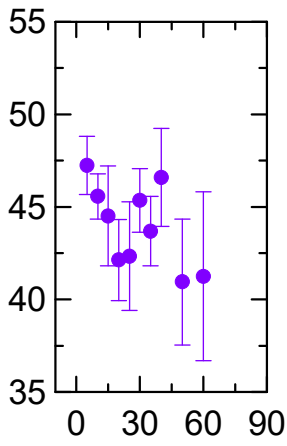

10

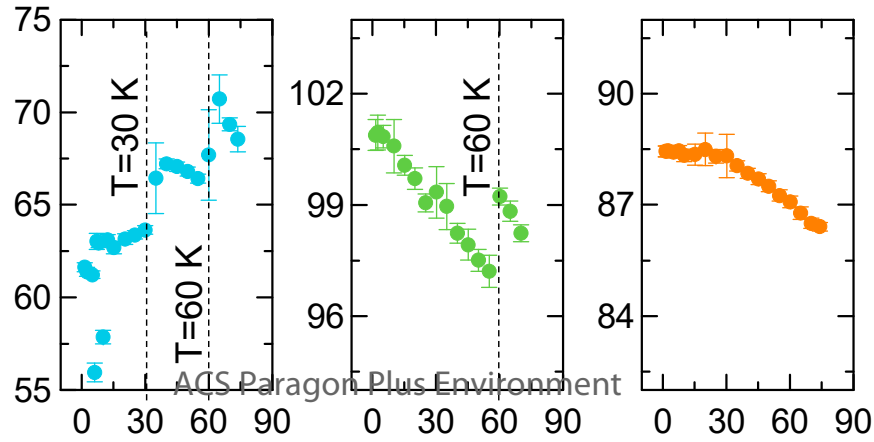

$\mathrm{T}(\mathrm{K})$

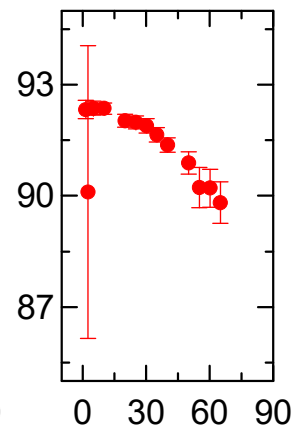




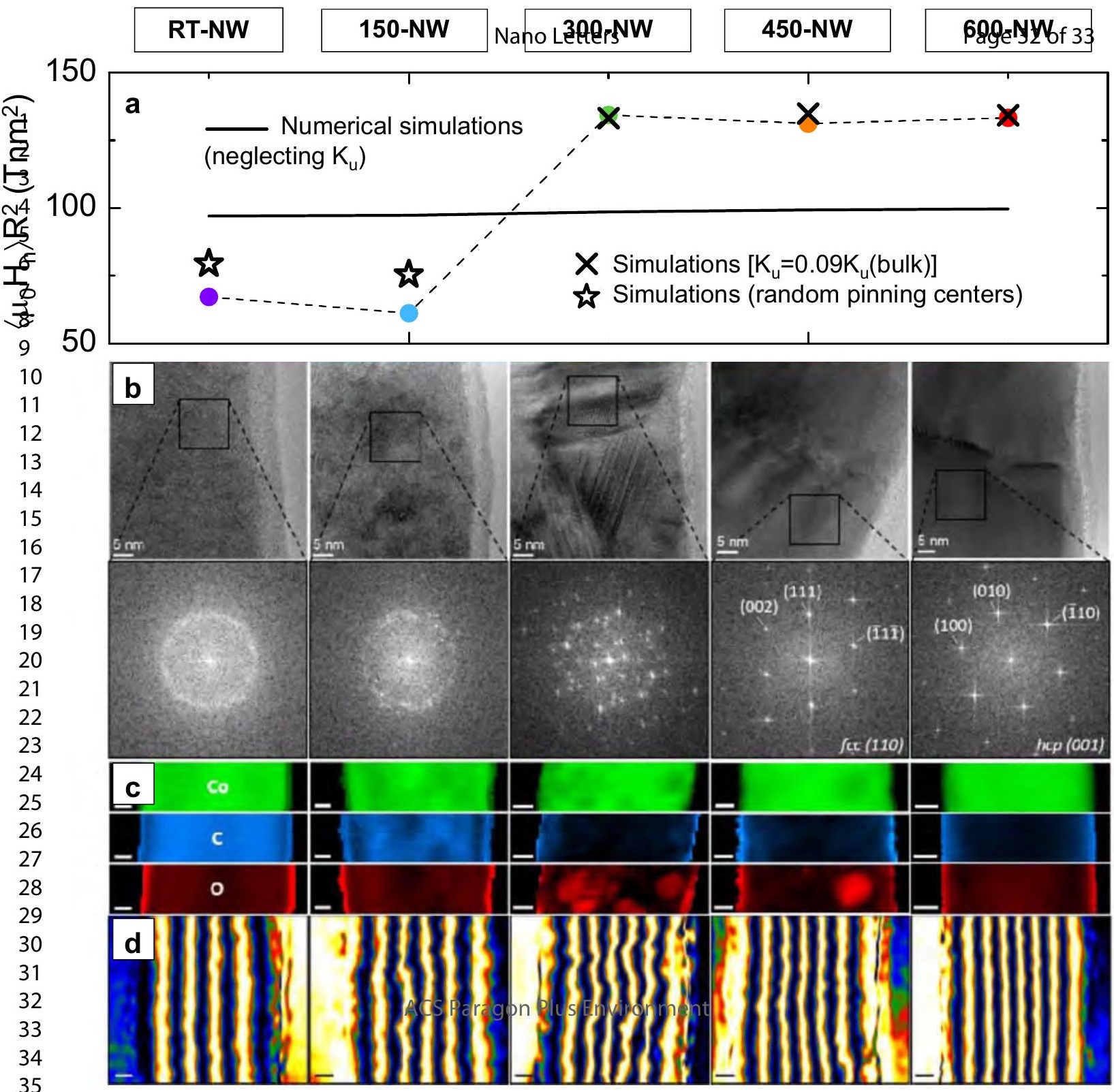




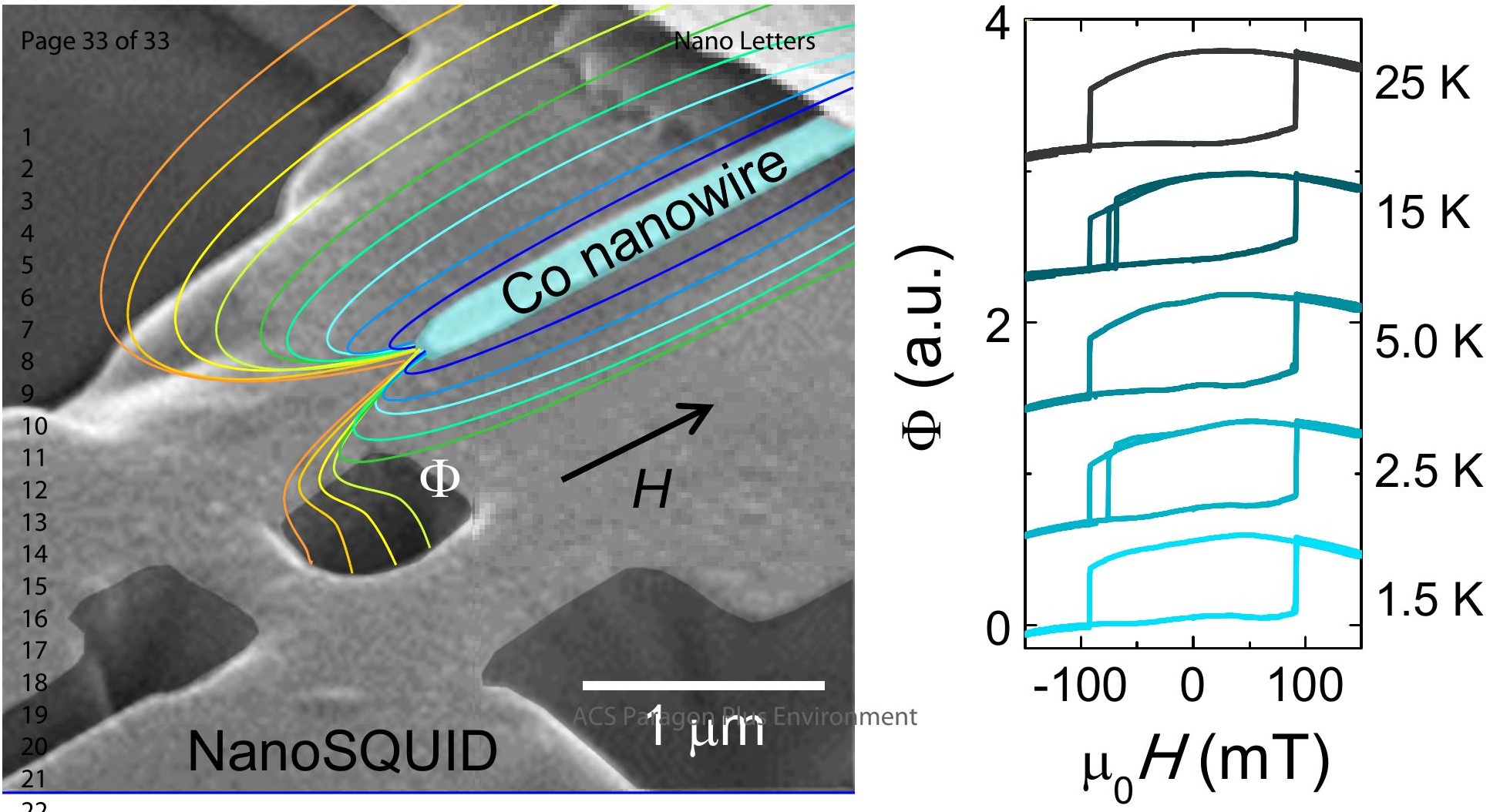

